\title{
(2) OPEN ACCESS \\ Fall-related deaths among older adults in British Columbia: cause and effect of policy change
}

\author{
Aayushi Joshi, ${ }^{1}$ Fahra Rajabali (1) , ${ }^{1}$ Kate Turcotte, ${ }^{1}$ M Denise Beaton, ${ }^{1,2}$ lan Pike ${ }^{1,3}$
}

${ }^{1} \mathrm{BC}$ Injury Research and Prevention Unit, BC Children's Hospital Research Institute, Vancouver, British Columbia, Canada

${ }^{2} \mathrm{BC}$ Centre for Disease Control, Provincial Health Services Authority, Vancouver, British Columbia, Canada

${ }^{3}$ Department of Pediatrics, UBC, Vancouver, British Columbia Canada

\section{Correspondence to}

Ms Fahra Rajabali, BC Injury Research and Prevention Unit, BC Children's Hospital Research Institute, Vancouver, BC V5Z 4H4, Canada; frajabali@bcchr. ca

Received 17 April 2019 Revised 19 July 2019 Accepted 24 July 2019 Published Online First 30 August 2019
ABSTRACT

Background The British Columbia Coroners

Service implemented a policy in 2010 advising the reclassification of underlying causes of deaths due to falls from 'natural' to 'accidental'. This study investigates whether observed data trends reflect this change in practice, are artefacts of inconsistent reporting, or indicate a true increase in fall-related deaths.

Methods Mortality data were analysed from 2004 to 2017 for cases with International Statistical Classification of Diseases and Related Health Problems, 10th Revision fall codes W00-W19, occurring among adults aged 60 years and older.

Results From 2010 to 2012, accidental fall-related deaths increased among those aged 80 years and older, followed by an increase in natural deaths with fall as the contributing cause.

Conclusions Changes in reporting resulting from the 2010 policy change were observed; however, post-2012 data indicate a reversion to previous reporting practices.

\section{INTRODUCTION}

Supporting the rapidly growing ageing population is a significant public health concern. Falls are the primary cause of injury-related deaths among community-dwelling older adults, with one in three falling each year. ${ }^{1}$ While the risk of falling increases with age, falls are not an inevitable part of ageing. Falls occur because of a complex interaction of multiple risk factors often categorised into four dimensions: biological, behavioural, environmental and socioeconomic. Increasing age often corresponds with increasing frailty, chronic medical conditions, and other individual and environmental variables. Falls can be prevented with evidencebased interventions, particularly those that address multifactorial risk factors. ${ }^{2-6}$ Fall prevention programming has become a focal public health issue among Canadian provinces and territories over the last decade, driven largely by mortality trends. In British Columbia (BC), there was no improvement in the rate of fall-related mortality between 1990 and 2001 among adults aged 65 years and older. ${ }^{7}$ From 1989 to 2006, all BC regional health authorities established fall prevention plans in response to this public health issue. ${ }^{8}$ In addition to local action, the international body of research regarding the effectiveness of fall prevention programming expanded rapidly during this period. Given the intersection of multiple risk factors, effective programme planning and evaluation depend on accurate reporting of fall-related mortality.
Medicolegal investigation and death classification remain integral to epidemiological research for accurate surveillance. Public health policy and prevention efforts rely on precise death classification and completion of death certificates as a method of reporting mortality information. ${ }^{9}{ }^{10}$ Death investigation in Canada is particularly heterogeneous; concerns have been raised about the quality of death investigations and integrity of mortality statistics in Canada due to a lack of overarching national authority for death investigation. ${ }^{9}$ All fall-related deaths in Canada include a contributing cause of death (CCD) coded for fall, including those where the main underlying cause of death (UCD) is also coded as an accidental death due to a fall. These terms are further explained in table $1 .^{11} 12$

In $\mathrm{BC}$, once a death certificate is signed by a certifier or coroner, it is sent to the BC Vital Statistics Agency (BCVS) for transcribing and coding. The World Health Organization's (WHO) International Statistical Classification of Diseases and Related Health Problems, 10th Revision (ICD-10) codes are assigned to categorise UCD and CCDs, and are used to describe trends and patterns for surveillance. Coding software at BCVS electronically transcribes information from the death certificate, followed by a manual review by Medical Coding Unit nurses to ensure accurate capture of information, with follow-up with the certifier as needed. ${ }^{13}$

While the BC Coroners Service (BCCS) does not assign codes, the BCCS Classification Guideline was revised on 1st September 2010 to improve identification of individuals whose deaths were related to a previous fall, bringing this in line with the change from the WHO International Statistical Classification of Diseases and Related Health Problems 9th Revision (ICD-9), to ICD-10 that occurred in the year 2000. The policy change is described as follows:

On September 1, 2010 the BCCS Classification Guideline was revised regarding deaths of persons who sustain injuries due to a fall, and whose health was compromised by significant pre-existing natural disease. These deaths were previously classified as natural, and are now classified as accidental. ${ }^{14}$

Physicians and nurse practitioners may certify only those deaths that are deemed natural. All other types of deaths, such as accidental, sudden and unexpected, unexplained, or unattended, are the responsibility of the BCCS, and undergo investigation. ${ }^{13}$ Older adults hospitalised from a fall-related injury may experience reduced mobility and failing health, eventually succumbing to other conditions, for example, pneumonia or cardiac arrest. Prior 
Table 1 Definition of terms for classifying deaths

(1) The disease or injury which initiated the train of morbid events leading directly to death or (2) The circumstances of the accident or

Underlying cause of death (UCD) Contributing cause of death (CCD) Natural death violence which produced the fatal injury. UCD can be classified as natural, accidental, homicide, suicide and undetermined. Other significant conditions contributing to the death, but not related to the disease or condition causing it.

A death primarily resulting from a disease of the body or progressive fatigue of bodily systems' A natural death does not result from injury or abnormal environmental factors.

A death primarily due to an event happening in an unexpected manner apart from planned human agency. to the year 2010, the BCCS classified these deaths as natural; following the 2010 policy change, they are now classified as accidental with the UCD specified as a fall, in line with ICD-10. ${ }^{15}$ To ensure accurate injury surveillance, it is necessary to monitor the effects of such a change in policy in order to understand how the policy may have an impact on the ongoing collection of data.

The objective of this study was to investigate an observed anomaly in fall-related mortality trends among older adults in $\mathrm{BC}$, and whether this anomaly is an artefact of reporting practices or indicates a true change in fall-related mortality rates.

\section{METHODS}

Mortality data from the BC Vital Statistics Agency (BCVS) were analysed for cases involving adults aged 60 years and older occurring from 2004 to 2017, with a fall indicated as a CCD (ICD-10 W00-W19). The data were divided to reflect those occurring prior to the policy change (2004-2009) and those occurring post-policy change (2011-2017), recognising that the data from 2010 represent the transition year. Of these cases where a fall was recognised as a contributing factor in the death, those identified as UCD 'accidental' (fall) versus UCD 'natural' were plotted as proportions and as mortality rates per 100000 population. Comparisons for UCD 'fall' cases were also made with falls mortality data from Alberta, Ontario, and Quebec, as well as national data. Age-specific mortality rates were calculated when comparing within and between age groups across years and provinces using the age-specific population from BCVS. Age-specific population was accessed from BC Stats. ${ }^{16}$

Data analyis was conducted using IBM SPSS Statstistics V.25.0. ${ }^{17}$ Time trends comparing the pre-policy and postpolicy change periods (2004-2009 vs 2011-2017) for the proportions of deaths and the mortality rates were explored by age group for UCD 'fall' versus UCD 'natural'. Significance testing using linear regression was conducted for each of the trend lines. The results were interpreted to be significant for $\mathrm{p}$ value $<0.05$.

\section{RESULTS}

A total of 13283 deaths occurred among adults aged 60 years and older in BC from 2004 to 2017, where fall was an identified CCD; 47\% $(n=6278)$ of cases captured the UCD as 'fall'. Prior to the policy change from 2004 to 2009 , there were a total of 5180 deaths where fall was an identified CCD; $36 \%$ of deaths were captured as UCD 'fall' $(n=1882)$, averaging 314 deaths per year. Following the policy change from 2011 to 2017, there were a total of 7163 deaths where fall was an identified CCD; 54\% of deaths were captured as UCD 'fall' $(n=3883)$, averaging 555 deaths per year. The remaining deaths occurred during 2010, the transition year (CCD 'fall' $n=940$, UCD 'fall' $n=513$ ).

The increase in the proportion of deaths coded as UCD 'fall' after the policy change (2011-2017) was highest among adults aged 80 years and older, comprising 78\% $(n=3029)$ of cases. A closer review of this age group showed a significant increase in the proportion of deaths captured as UCD 'fall' versus UCD 'natural' after 2008 until 2012 (p<0.001), after which the numbers showed a significant gradual decrease to 2017 ( $p<0.001$ ) (figure 1). The mirrored pattern of proportions was observed for falls captured as UCD 'natural', with a significant upward trend from 2012 to 2017 ( $\mathrm{p}<0.001$ ) (figure 1). Similar trends were seen when the deaths were depicted as rates (figure 2), although there was a sharp, significant upward trend in the mortality rates for falls captured as UCD 'natural' from 2015 to 2017 ( $\mathrm{p}<0.001)$. Overall, the mortality rates per 100000 population for all fall-related deaths identified by CCD during this period of transition were stable with a non-significant trend observed: 432.6 in 2008, 440.7 in 2009, 439.8 in 2010 and 431.0 in 2011 (figure 2).

Similar analyses of age groups 60-69 and 70-79 years were conducted. Among adults aged 60-69 years, the majority of fallrelated deaths were captured as UCD 'fall', increasing slightly with the policy change, and significantly decreasing again from 2014 to 2017 ( $p<0.001$ ), while UCD 'natural' cases had a significant increase $(\mathrm{p}<0.001)$ (figure 3$)$. Annual fall-related mortality rates did not change significantly for this age group from 2009 to 2017 (figure 4).

Among adults aged 70-79 years, the proportion of fall-related deaths captured as UCD 'fall' showed a significant increase from 2008 to $2011(\mathrm{p}<0.001)$, followed by a significant gradual descent from 2012 to $2017(\mathrm{p}<0.001)$ (figure 3). Trends for mortality rates among those aged 70-79 depicted a sharp increase for fall-related deaths captured as UCD 'fall' immediately following the policy change until 2011, after which the rates showed a downward non-significant trend from 2012 to 2017 (figure 4). The annual mortality rates per 100000 population (2008-2011) for all fall-related deaths identified by CCD among ages 70-79 were stable with a non-significant trend observed: 55.5 in 2008, 54.2 in 2009, 40.4 in 2010 and 49.8 in 2011 (figure 4).

Compared with the other provinces, a different pattern was seen in the trend of UCD 'fall' death rates among adults aged 80

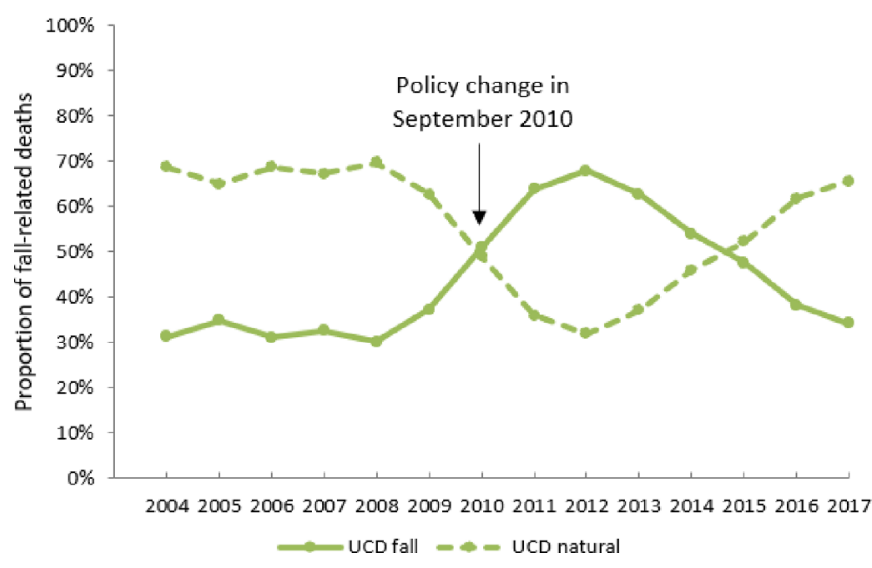

Figure 1 Proportion of fall-related deaths among adults 80 years and older, by UCD, British Columbia, 2004-2017. UCD, underlying cause of death. 


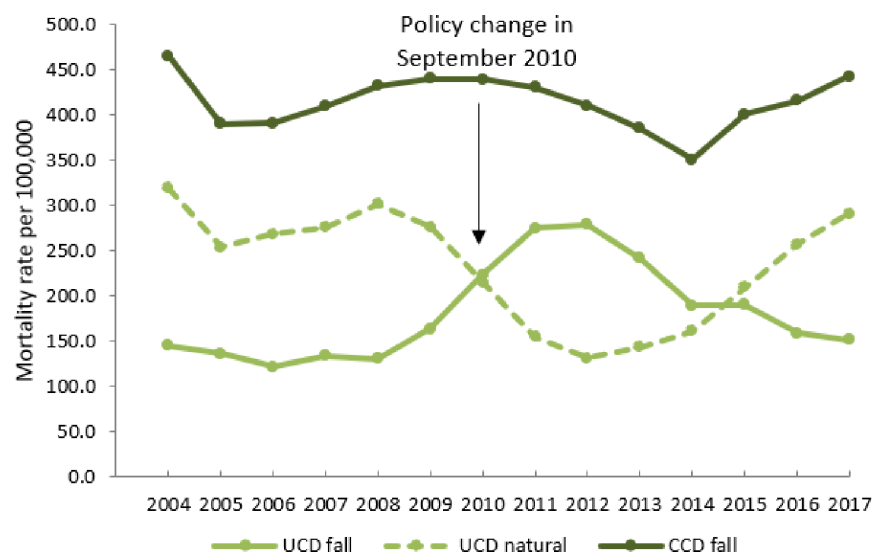

Figure 2 Fall-related mortality rates per 100000 population among adults 80 years and older, by cause of death, British Columbia, 20042017. CCD, contributing cause of death; UCD, underlying cause of death.

and older in BC, peaking in $2012(\mathrm{p}<0.01)$, followed by a significant decrease in rates to $2017(\mathrm{p}<0.05)$ (figure 5). The national trend was higher than both Alberta and Quebec.

\section{DISCUSSION}

The increase in both the proportions and rates of deaths as resulting from a fall rather than from natural causes among those aged 80 years and older is indicative of the impact of the $2010 \mathrm{BC}$ Coroners falls mortality reporting policy change. Discussions and calls for fall-related mortality cases to be classified as 'accidental' rather than 'natural', complying with ICD-10 requirements, may have influenced practices leading up to the policy change. Increases in the proportion of all fall-related deaths captured as UCD 'fall' were found to be significant after 2008 until 2012. This observed increase appears to be related to the policy change rather than a true increase in fall-related mortality rates, as the fall-related mortality rates for CCD 'fall' cases do not significantly increase during this period. As there were no subsequent classification guideline updates after 2010, the proportion of cases with a UCD 'fall' as compared with UCD 'natural' might be expected to remain high beyond 2012. However, among those aged 80 years and older, there was a reverse in the trend, with the proportion of UCD 'fall' cases steadily declining from 2012 to 2017. This trend may indicate varying reporting practice by certifiers or coroners in combination with an unofficial reversion to previous reporting practices. Interestingly,

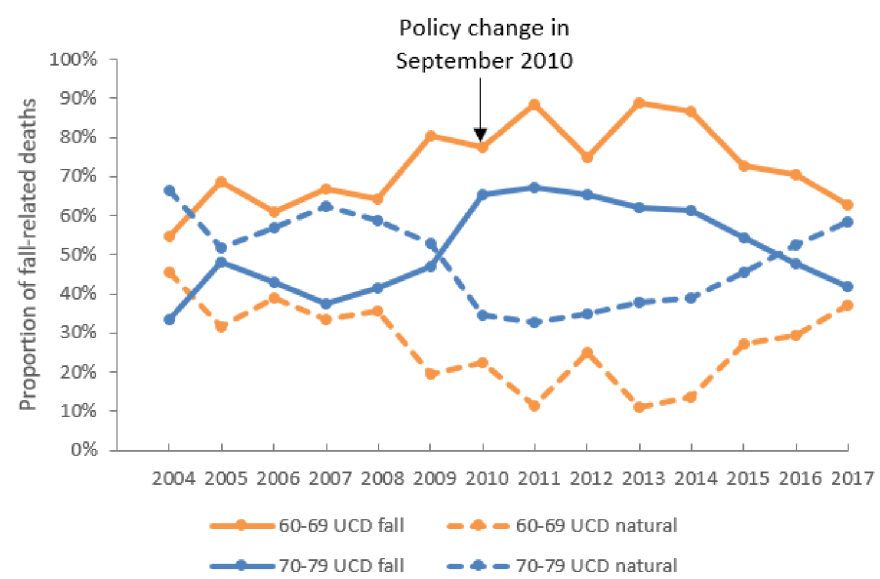

Figure 3 Proportion of fall-related deaths among older adults aged 60-69 and 70-79, by UCD, British Columbia, 2004-2017. UCD, underlying cause of death.

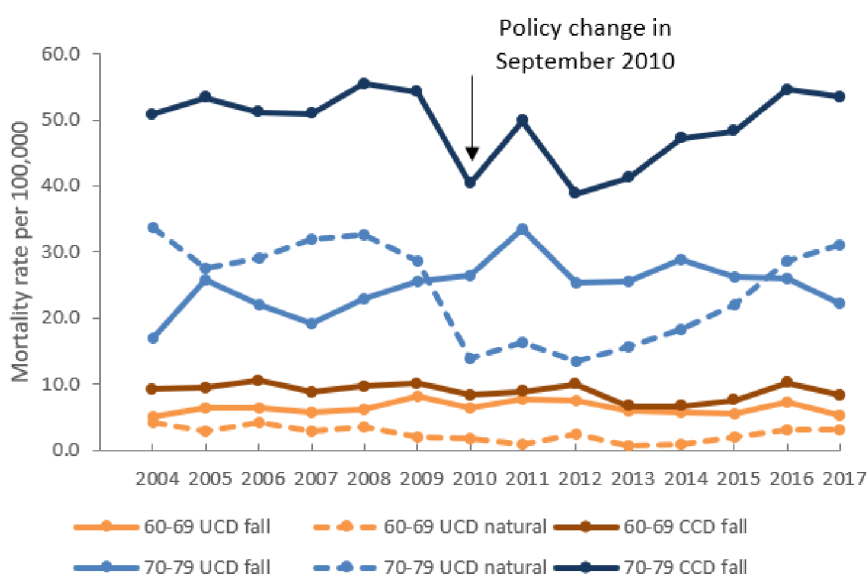

Figure 4 Fall-related mortality rates per 100000 population among older adults aged $60-69$ and $70-79$, by cause of death, British Columbia, 2004-2017. CCD, contributing cause of death; UCD, underlying cause of death.

among those 60-69 years, the policy may have had a slight influence in classification practices, yet the proportions of UCD 'fall' remained consistently high during the study period. Among those $70-79$ years, a trend similar to that of those 80 years and older is seen, with the proportion of UCD 'fall' increasing in relation to the policy change rather than a true increase in fall-related deaths, and slowly reverting back to prepolicy levels.

Misidentification of fall-related mortality as death due to natural causes rather than to accidental causes leads to an underestimate of the true burden of falls among older adults, while the converse results in an overestimate, and cause for alarm. These findings indicate that the true rate of fall-related mortality among older adults in $\mathrm{BC}$ is sensitive to classification practices and was masked by the 2010 policy change. These findings are supported by provincial comparisons, with Alberta and Quebec demonstrating relatively stable mortality rates during the same time period, while rates in BC between 2010 and 2013 were seen to fluctuate significantly year to year, with a downward trend after 2013. Such variability in reporting raises uncertainty as to the true levels of older adults' fallrelated mortality in BC. Is fall-related mortality among older adults truly decreasing or is this an artefact of an unofficial reversion to pre-2010 classification practices?

The standardisation of death reporting is an important task. In recognition of this, the BCCS supports the improvement of accurate death certificate reporting through newsletters disseminated to the medical community and to community care facilities, in

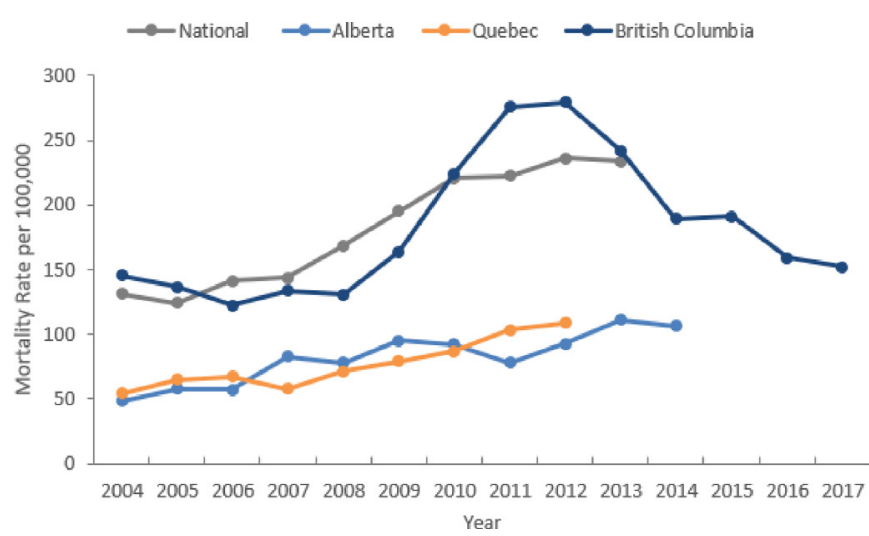

Figure 5 Fall-related mortality rates among older adults aged 80 and older, national and by province, 2004-2017. 
order to educate certifiers. Occasionally, BCVS may resubmit cases to be considered for a coroner review in order to ensure accurate coding of all deaths. One result of the 2010 policy change was an increase in the proportion of CCD 'fall' deaths classified as UCD 'fall', thereby increasing the number of deaths requiring a coroner review. This placed an increased burden on the coronial system and on the families of the deceased to investigate these deaths, particularly for ages 80 years and older. However, it is also possible that the increased burden, combined with the perception of falls among the oldest old as inevitable, influenced a shift away from the more stringent use of the UCD 'fall' coding seen immediately post policy change.

To date, there are no previous studies investigating the influence of a lack of national medicolegal system coding standards, and the resulting provincial and territorial variation in quality of falls mortality statistics. The medical examiner system is used in four provinces (Alberta, Manitoba, Nova Scotia, Newfoundland and Labrador), whereas the rest of the country operates under a coronial system. ${ }^{18}$ Medical examiners are required to be qualified in pathology in addition to being licensed physicians; however, a coroner may be a licensed physician, lawyer or lay person, although they are often in the medical profession. ${ }^{18}$ Unlike in Ontario, where all coroners are physicians, coroners in $\mathrm{BC}$ have a variety of backgrounds, and a judge may perform the duties of a coroner at the request of the Attorney General. ${ }^{19}$

This heterogeneity, coupled with a lack of national standards, training, credentialing, or agreement on evaluation indicators, creates substantial variation in death classification practices across Canada. ${ }^{9}$ An investigation into the validity of death certification by 226 Ontario coroners revealed under-reporting of accidental deaths involving an injury that resulted in a natural complication, where there was a delay between injury and death. A discrepancy was found between types of death, wherein deaths due to injuries from an overdose had decreased odds of being accurately reported as compared with deaths due to drowning, hanging or carbon monoxide poisoning. ${ }^{20}$

In the USA, there is significant variance between state-specific fall mortality rates among adults 65 years and older ranging from 13.9 to 140.4 per $100000 .{ }^{21}$ Based on a forensic review of hospital discharge data, Koehler et al concluded that the manner of death for 12 of 34 natural deaths involving a fall should be changed from natural death to accidental fall, ${ }^{22}$ while an Idaho study found that non-physician coroners had lower accuracy rates compared with physicians when certifying cancer deaths. ${ }^{23}$ An audit of 1000 death certificates in the UK revealed that $55 \%$ were completed to a minimally accepted standard, many without sufficient information to allow for correct ICD-10 coding, and approximately 10\% deemed illogical or inaccurately completed. ${ }^{24}$ Studdert and Cordner in Australia assessed the impact of coronial investigations from 2000 to 2007 , where a change in manner of death or in intent classification occurred in only $5.2 \%$ of cases. ${ }^{25}$

According to current literature, mortality data do not appear to identify all instances of fall-related deaths. ${ }^{20-22} 25$ Undercounting of fall-related deaths is often due to a failure to acknowledge the significance of a fall event that led to medical complications and death. Understanding the types of deaths most likely to be misclassified, as well as how they are misclassified, allows greater insight into improving death certification training and further research to more accurately assess accidental deaths. In a report on the comparability of cause of death between ICD-9 and ICD-10, select changes in coding instructions were found to have the greatest impact on mortality data, often affecting cases involving pneumonia. ${ }^{15}$ In ICD-10, the recognition that pneumonia is often the result of another condition or injury led to it being less likely selected as the
UCD, as compared with ICD-9. ICD-10 allows for the classification of the unintentional injury as the UCD for injuries occurring within 1 year of death, while under ICD-9, the injury had to occur within 4 weeks prior to death. ${ }^{15}$

The most common recommendation to improve the quality of mortality databases and completion of death certification is mandatory training of physicians, nurse practitioners and coroners in the completion of proper death certification. ${ }^{921} 22$ The WHO provides an instruction manual that describes in detail how to certify a death according to ICD-10. ${ }^{26}$ Other suggestions include regular consultation with pathologists and the development of national death certification coding practices. ${ }^{9212324}$ Considering the complex, multifactorial nature of fall-related deaths among older adults, using a multiple cause analysis, wherein all conditions on the death certificate and reports are recognised, would provide a clearer picture of the true burden of fall-related deaths and improve surveillance accuracy. To ensure fluency among all users, training on the forthcoming adjustment to ICD-11 should be emphasised, and the complex ways in which the use of databases is shaped by local context over time should be recognised.

Understanding how policies can influence data capture and reporting is critical to setting goals and benchmarks for prevention and subsequent surveillance. Advocates within the public health system were vocal supporters of the BCCS 2010 policy change in capturing fall-related deaths to align with ICD-10 classification. However, the apparent reversion in practice during the longerterm postpolicy period identifies the need for continuous quality monitoring to ensure accurate data, which in turn informs effective and efficient resource use for public health fall prevention programming. Performance measures and clear baselines are key in planning future work and evaluating present efforts. The performance measures for injury prevention in Promote, Protect, Prevent - BC's Guiding Framework for Public Health and Healthy Families BC Policy Framework includes the age-standardised mortality rate for unintentional injuries for British Columbians. The baseline for this performance measure was set using 2010 data at 25.5 per 100000 population, with a 2023 target of 15 per 100000 population. ${ }^{27}$

The present study shows that using 2010 as a baseline year misrepresented the actual picture of fall-related mortality due to the policy change, potentially inflating the baseline measure for age-standardised mortality rate for unintentional injuries for British Columbians. If 2008 is viewed as the baseline year and compared with 2017, fall-related mortality remained relatively stable among older adults aged 65 years and above from 47.6 per 100000 population in 2008 to 51.5 per 100000 in 2017, a nonsignificant trend. ${ }^{28}$ While the efforts in $\mathrm{BC}$ have primarily focused on acute and long-term care settings, the majority of falls among older adults occur within community. In order to reduce the burden of falls among community-dwelling older adults, further multifactorial action is needed to bolster existing programming in $\mathrm{BC}$, extending it beyond acute and long-term care settings.

\section{Data limitations}

This study is based on a dataset of older adult deaths that included a CCD for fall. All cases with a UCD fall will also have a CCD fall, whereas only a subset of cases with UCD natural will have a CCD fall. Therefore, it is likely that there may be UCD natural cases where a fall was involved, but do not include a CCD for fall, and therefore have not been included in this analysis.

\section{CONCLUSION}

Data influence public health policy, practice and evaluation. This study demonstrates that policy and practice can also have 
a significant influence on data. The 2010 BCCS policy change, aligning with ICD-10 coding, was intended to highlight the true burden of falls among older adults. The increased mortality rates and associated burden on the coronial system and the families of the deceased following this policy change appear to have prompted an undocumented reversion of coding practices. This trend was not mirrored in other Canadian provinces. Without an understanding of why fall-related mortality rates escalated in $\mathrm{BC}$ over this time period, it may be misinterpreted that fall-related deaths among older adults were increasing, particularly among those 80 years and older, despite fall prevention programming across the province. Further, there may be value in considering multiple-cause analysis for fall-related mortality among older adults, to accurately inform data surveillance. This would provide a foundation for fall prevention programming and evaluation to reduce the burden of falls among community-dwelling older adults.

\section{What is already known on the subject}

- Falls are the leading cause of injury-related mortality among older adults, with one in three older adults in Canada falling each year.

- Public health interventions to prevent falls and fall-related injuries rely in part on mortality data to set benchmarks and goals, monitor progress and evaluate the impact of an intervention, and inform public policy.

- The British Columbia Coroners Service (BCCS) Classification Guideline was revised on 1 September 2010 to improve identification of deaths related to a previous fall.

\section{What this study adds}

- The BCCS policy change influenced observed time trends in fall-related mortality for older adults in $B C$, and therefore $B C$ data skewed national data.

- The observed time trends in fall-related mortality for older adults in $\mathrm{BC}$ do not represent a true increase in rates.

- To date, this is the first study investigating the influence of a lack of national standards, and the resulting variation across provinces and territories in Canada's medicolegal system, on the quality of national mortality statistics.

Funding statement The authors have not declared a specific grant for this research from any funding agency in the public, commercial or not-for-profit sectors.

Competing interests None declared.

Patient consent for publication Not required.

Ethics approval This study received ethics approval from the University of British Columbia Children's \& Women's Health Centre of British Columbia Research Ethics Board (cert number CW17-0108/H17-00859).

Provenance and peer review Not commissioned; externally peer reviewed.

Open access This is an open access article distributed in accordance with the Creative Commons Attribution Non Commercial (CC BY-NC 4.0) license, which permits others to distribute, remix, adapt, build upon this work non-commercially, and license their derivative works on different terms, provided the original work is properly cited, appropriate credit is given, any changes made indicated, and the use is non-commercial. See: http://creativecommons.org/licenses/by-nc/4.0/.

ORCID iD

Fahra Rajabali http://orcid.org/0000-0002-2990-6151

\section{REFERENCES}

1. Public Health Agency of Canada. Seniors' Falls in Canada: Second Report, 2014. Available: http://epe.lac-bac.gc.ca/100/201/301/weekly_checklist/2014/internet/w1436-U-E.html/collections/collection_2014/aspc-phac/HP25-1-2014-eng.pdf [Accessed 7 Feb 2019].

2. Beard J, Rowell D, Scott D, et al. Economic analysis of a community-based falls prevention program. Public Health 2006;120:742-51.

3. Masters R, Anwar E, Collins B, et al. Return on investment of public health interventions: a systematic review. J Epidemiol Community Health 2017;71:827-34

4. Towne SD, Li Y, Lee S, et al. Physical activity and associated medical cost savings among at-risk older adults participating a community-based health \& wellness program. PloS One 2018;13:e0198239-19.

5. Grossman DC, Curry SJ, Owens DK, et al. Interventions to prevent falls in communitydwelling older adults: US preventive services Task force recommendation statement. JAMA 2018;319:1696-704.

6. Phelan EA, Ritchey K. Fall prevention in community-dwelling older adults. Ann Intern Med 2018;169:ITC81-ITC94.

7. Scott V, Peck S, Kendall P, BC Ministry of Health Planning. Prevention of falls and injuries among the elderly: a special report from the office of the provincial health officer., 2004. Available: https://www.health.gov.bc.ca/library/publications/year/2004/ falls.pdf [Accessed 17 Apr 2019].

8. Scott $\mathrm{V}$, Herman M, Gallagher E.The evolution of falls and injury prevention among seniors in British Columbia, Canada. Open Longevity Sci; 2011: 16-25.

9. Kelsall D, Bowes MJ. No standards: medicolegal investigation of deaths. CMAJ 2016;188:169.

10. Maudsley G, Williams EM. "Inaccuracy' in death certification--where are we now? J Public Health Med 1996;18:59-66. Available.

11. Dominion Bureau of Statistics, Ottawa. Vital statistics Handbook methods., 1957. Available: http://publications.gc.ca/collections/collection_2017/statcan/CS12-5091957-eng.pdf [Accessed 17 April 2019].

12. British Columbia Vital Statistics Agency. Physicians' and coroners' handbook on medical certification of death and stillbirth, 2014. Available: http://unstats.un.org/ unsd/vitalstatkb/Attachment32.aspx?AttachmentType $=1$

13. Vital Statistics Act, [RSBC 1996] Chapter 479. Available: http://www.bclaws.ca/Recon/ document/ID/freeside/00_96479_01

14. BC Coroners Service. BC Coroners Service 2010 annual report; 2013: 1-101. accessed 1 May 2018 https://www2.gov.bc.ca/assets/gov/birth-adoption-death-marriage-anddivorce/deaths/coroners-service/reports/annual/2010.pdf

15. Anderson RN, Miniño AM, et al, Hyattsville, Maryland: National Center for Health Statistics. Comparability of cause of death between ICD-9 and ICD-10: preliminary estimates. National Vital Statistics Report 2001;49:1-32.

16 BC Stats, Population estimates. Available: https://www2.gov.bc.ca/gov/content/data/ statistics/people-population-community/population/population-estimates [Accessed 31 October 2018].

17 IBM Corporation: SPSS statistics for windows version 25. Act C. [SBC 2007] Chapter 15, 2017. Available: http://www.bclaws.ca/civix/document/id/ complete/ statreg/07015 01 [Accessed 10 Apr 2018].

18. Avis SP. Death investigation in Canada. J Forensic Sci 1998;43:377-9.

19 BC Coroners Act. [SBC 2007] Chapter 15. Available: http://www.bclaws.ca/civix/ document/id/complete/statreg/07015_01 [Accessed 10 Apr 2018].

20. Parai JL, Kreiger N, Tomlinson G, et al. The validity of the certification of manner of death by Ontario coroners. Ann Epidemiol 2006;16:805-11.

21. Maresh J, Guse C, Layde P. National trends and coding patterns in fall-related mortality among the elderly in the United States. J Public Health Policy 2012;33:202-14.

22. Koehler SA, Weiss HB, Shakir A, et al. Accurately assessing elderly fall deaths using hospital discharge and vital statistics data. Am J Forensic Med Pathol 2006;27:30-5.

23. Johnson CJ, Hahn CG, Fink AK, et al. Variability in cancer death certificate accuracy by characteristics of death certifiers. Am J Forensic Med Pathol 2012;33:137-42.

24. Swift B, West K. Death certification: an audit of practice entering the 21 st century. J Clin Pathol 2002;55:275-9.

25. Studdert DM, Cordner SM. Impact of coronial investigations on manner and cause of death determinations in Australia, 2000-2007. MJA 2010;192

26. World Health Organization. World Health Organization International Statistical Classification of Diseases and Related Health Problems 10th (ICD-10). Available: https://icd.who.int/browse10/Content/statichtml/ICD10Volume2 en_2016.pdf

27. Ministry of health. Promote, protect, prevent: Our health begins here. $B C^{\prime}$ 's guiding framework for public health (Update 2017): Victoria, BC, 2017. Available: https:// www.health.gov.bc.ca/library/publications/year/2017/BC-guiding-framework-forpublic-health-2017-update.pdf [Accessed 17 Apr 2019].

28. BC Vital Statistics. Fall-Related fatality data 2008-2017 (accessed from bc centre of disease control, chronic disease and injury Datamart); 2019 [accessed 17 Jan 2019]. 